\title{
Modeling of Schottky Barrier Diode Millimeter-Wave Multipliers at Cryogenic Temperatures
}

Johansen, Tom K.; Rybalko, Oleksandr; Zhurbenko, Vitaliy; Bowen, Sean; Hesler, Jeffrey; ArdenkjærLarsen, Jan Henrik

Published in:

Proceedings. 2015 SBMO/IEEE MTT-S International Microwave and Optoelectronics Conference (IMOC)

Link to article, DOI:

10.1109/IMOC.2015.7369088

Publication date:

2015

Document Version

Peer reviewed version

Link back to DTU Orbit

Citation (APA):

Johansen, T. K., Rybalko, O., Zhurbenko, V., Bowen, S., Hesler, J., \& Ardenkjær-Larsen, J. H. (2015). Modeling of Schottky Barrier Diode Millimeter-Wave Multipliers at Cryogenic Temperatures. In Proceedings. 2015 SBMO/IEEE MTT-S International Microwave and Optoelectronics Conference (IMOC) IEEE. https://doi.org/10.1109/IMOC.2015.7369088

\section{General rights}

Copyright and moral rights for the publications made accessible in the public portal are retained by the authors and/or other copyright owners and it is a condition of accessing publications that users recognise and abide by the legal requirements associated with these rights.

- Users may download and print one copy of any publication from the public portal for the purpose of private study or research.

- You may not further distribute the material or use it for any profit-making activity or commercial gain

- You may freely distribute the URL identifying the publication in the public portal 


\title{
Modeling of Schottky Barrier Diode Millimeter-Wave Multipliers at Cryogenic Temperatures
}

\author{
Tom K. Johansen ${ }^{1}$, Oleksandr Rybalko ${ }^{1}$, Vitaliy Zhurbenko ${ }^{1}$, Sean Bowen ${ }^{1}$, Jeffrey Hesler ${ }^{2}$, \\ Jan H. Ardenkjaer-Larsen ${ }^{1}$ \\ ${ }^{1}$ Department of Electrical Engineering \\ Technical University of Denmark \\ Kgs. Lyngby, Denmark \\ tkj/rybalko/vz/bowen/jhar@elektro.dtu.dk \\ ${ }^{2}$ Virginia Diodes Inc. \\ Charlottesville, VA 22902 USA \\ hesler@vadides.com
}

\begin{abstract}
We report on the evaluation of Schottky barrier diode GaAs multipliers at cryogenic temperatures. A GaAs Schottky barrier diode model is developed for theoretical estimation of doubler performance. The model is used to predict efficiency of doublers from room to cryogenic temperatures. The theoretical estimation is verified experimentally using a $78 \mathrm{GHz}$ doubler cooled down to $14 \mathrm{~K}$. The observed efficiency improvement due to cooling is approximately $4 \%$ per 100 degrees.
\end{abstract}

Keywords - cryogenic temperature; frequency multiplier; GaAs diodes; millimeter wave; varactor.

\section{INTRODUCTION}

Varactor frequency multipliers are often used as solid-state sources of millimeter and submillimeter wave radiation in dynamic nuclear polarization systems [1]. The efficiency of polarization in such systems depends on power of electromagnetic radiation delivered to the sample. Traditionally, this power is delivered from sources using long wave-guiding structures, which loss and price scales with operating frequency. Alternatively, the multiplier can be placed close to the sample inside the polarization system, where the temperature of the environment is in the range from 77 to $100 \mathrm{~K}$. Usually, the performance of commercially available multipliers are not specified at such temperatures. The application, however, calls for theoretical and practical evaluation of the multiplier behavior in cryogenic environment. This work investigates the efficiency of Schottky barrier diode GaAs multipliers in the range of temperatures from $14 \mathrm{~K}$ to $300 \mathrm{~K}$. First, a model of the diode is developed. The model is then used to predict multiplier efficiency using harmonic balance analysis. The obtained data is compared with experimental results taken from the literature.

Compact diode models are available in most RF and microwave CAD software packages. Such diode models are generic, representing mainly the physical behavior of Silicon pn-junction diodes. Embedded with parasitic networks these diode models can also be used to describe the performance of room temperature Schottky barrier diode multipliers and mixers operating at frequencies up to several hundreds of $\mathrm{GHz}$ [2]. The temperature dependence of a pn-junction diode, however, is quite different from that of a Schottky barrier diode due to the different carrier transport mechanisms involved [3]. The physical based Schottky barrier diode model recently developed at the Technical University of Denmark and Goethe University of Frankfurt am Main [4] should in principle be able to predict the temperature behavior at cryogenic temperatures. The physical diode model, however, requires detailed knowledge about the technology used to fabricate the device. Typically, such detailed knowledge is unavailable to circuit designers and models must be based on a few parameters extracted from measured $\mathrm{I}-\mathrm{V}$ and $\mathrm{C}-\mathrm{V}$ characteristics on fabricated devices.

The following Section of the paper is dedicated to the development of an electro-thermal diode model, which allows the prediction of the performance of GaAs schottky barrier diode multipliers even at cryogenic temperatures. A simulation setup to test the performance of doublers at cryogenic temperatures is discussed in Section III. The results of the analysis are compared with the measured data for a millimeter-wave doubler in Section IV.

\section{SCHOTTKY BARRIER DIODE MODELING}

The schematic representation of the electro-thermal Schottky barrier diode model is shown in Fig. 1. It is implemented as a symbolic defined device (SDD) in Agilent ADS2013. In the electrical model the Schottky barrier diode is modelled by a nonlinear current source, $I_{d}$, nonlinear charge source, $Q_{d}$, and series resistance $R_{s}$. The model includes only the intrinsic part of the diode. Any external parasitic elements are assumed to be absorbed into the diode's embedded impedances. Also, the effect of semiconductor carrier-inertia, displacement current and skin-effect on the series resistance is at present not included in the model. This limits the application of the shown model to frequencies in the millimeter-wave frequency range. The electrical parameters are made dependent on the temperature by considering the physical properties of the GaAs Schottky barrier diode as explained later. The temperature rise, $\delta T_{d}$, due to self-heating 
in the device is related to the dissipated power $p(t)$ by an equivalent first-order network in the thermal model. The thermal resistance is taken independent of temperature despite the well-known dependence of the thermal conductivity of GaAs on temperature. This is done for better convergence during circuit simulations.

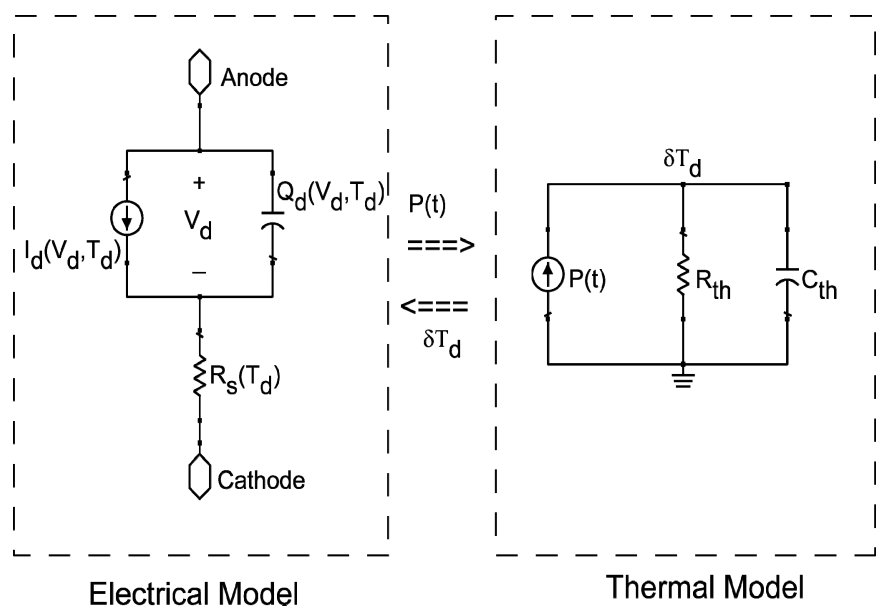

Fig. 1. Schematic representation of electro-thermal Schottky barrier diode model implemented as a SDD in Agilent ADS2013.

The temperature dependent I-V characteristic of a Schottky barrier diode is given by [5]

$$
I_{d}\left(V_{d}, T_{d}\right)=A R^{* *} \theta\left(T_{d}\right)^{2} \exp \left(\frac{-q \phi_{b}}{k_{B} \theta\left(T_{d}\right)}\right)\left(\exp \left(\frac{q V_{d}}{k_{B} \theta\left(T_{d}\right)}\right)-1\right)
$$

where $A$ is the anode area, $R^{* *}$ is the modified Richardson's constant, $T_{d}$ is the absolute temperature, $b$ is the barrier height, $q$ the electron charge, and $k_{B}$ is Boltzmann's constant. The temperature dependence is contained in the so-called slope parameter:

$$
\theta\left(T_{d}\right)=\theta_{f} \operatorname{coth}\left(\frac{\theta_{f}}{T_{d}}\right)
$$

with

$$
\theta_{f} \approx \alpha \frac{q \hbar}{k_{B}} \sqrt{\frac{N_{d}}{4 \varepsilon_{s} m^{*}}}
$$

where $\hbar$ is Plank's constant divided by $2 \pi, N_{d}$ is the doping of the epi-layer, $\varepsilon_{s}$ is the permittivity of the semiconductor, and $m^{*}$ is the effective mass of electrons in the conduction band. Physically, the I-V characteristic described by equations (1), (2) and (3) connects the limiting cases of current transport at very low temperatures where tunneling through the barrier dominates, with thermionic emission that dominates at temperatures above approximately $100 \mathrm{~K}$ [6]. The slope parameter is introduced in a way as to take into account of contributions from transport mechanisms other than thermionic emission also at higher temperatures. It is interesting to note that for a given semiconductor material, the only two unknown parameters determining the temperature dependence of the I-V characteristic are the barrier height and doping of the epi-layer. The leading factor, $\alpha$, in equation (3), however, models an effective epilayer doping which is experimentally observed to be higher than the nominal $N_{d}$ [6]. The representation of (1) as implemented in the SDD model is

$$
I_{d}\left(V_{d}, T_{d}\right)=I_{s}\left(T_{d}\right)\left(\exp \left(\frac{q V_{d}}{\eta\left(T_{d}\right) k_{B} T_{d}}\right)-1\right)
$$

where the saturation current $I_{s}$ and ideality factor $\eta$ have been introduced. The temperature dependence of the saturation current is evaluated as:

$$
I_{s}\left(T_{d}\right)=I_{s}\left(T_{\text {nom }}\right)\left(\frac{\theta\left(T_{d}\right)}{\theta\left(T_{\text {nom }}\right)}\right)^{2} \exp \left(\frac{q \phi_{B}}{k_{B}}\left(\frac{1}{\theta\left(T_{\text {nom }}\right)}-\frac{1}{\theta\left(T_{d}\right)}\right)\right)
$$

with $I_{S}\left(T_{\text {nom }}\right)$ representing the saturation current extracted from the measured I-V characteristic at the nominal temperature $T_{\text {nom }}$. Similar the temperature dependent ideality factor is evaluated as:

$$
\eta\left(T_{d}\right)=\eta\left(T_{\text {nom }}\right) \frac{\theta\left(T_{d}\right)}{T_{d}} \frac{T_{\text {nom }}}{\theta\left(T_{\text {nom }}\right)}
$$

where $\eta\left(T_{\text {nom }}\right)$ represents the ideality factor extracted from the measured I-V characteristic at the temperature $T_{\text {nom}}$.

The depletion capacitance of the Schottky barrier diode is modeled as [3]

$$
C_{j}\left(V_{d}, T_{d}\right)=\frac{C_{j o}}{\sqrt{1-\frac{V_{d}}{V_{b i}\left(T_{d}\right)-\frac{k_{B} T_{d}}{q}}}}
$$

where $C_{j o}$ is the zero bias junction capacitance extracted from $\mathrm{C}-\mathrm{V}$ measurements and $V_{b i}$ is the built-in potential. The builtin potential is related to the barrier height and epi-layer doping through [3]

$$
V_{b i}\left(T_{d}\right)=\phi_{b}-\frac{k_{B} T_{d}}{q} \ln \left(\frac{N_{c}}{N_{d}}\right)
$$

where $N_{c}$ is the effective density of states in the conduction band of GaAs. The singularity at high forward bias in (7) is avoided by a linear extrapolation of the depletion capacitance above the voltage $F_{c} V_{d}$ where $F_{c}$ is a factor between 0 and 1 .

The series resistance $R_{\mathrm{s}}$ of the Schottky barrier model contains three contributions; the resistance of the undepleted part of the epi-layer, the spreading resistance of the heavily doped substrate layer and the contact resistance. It is assumed that only the epi-layer resistance varies with temperature. In the developed SDD model the total series resistance is partitioned into two parts by using the parameter $X_{r}$ according to [7]

$$
R_{s}\left(T_{d}\right)=X_{r} R_{s}\left(T_{n o m}\right)\left(\frac{T_{d}}{T_{\text {nот }}}\right)^{0.89}+\left(1-X_{r}\right) R_{s}\left(T_{\text {nom }}\right)
$$

where the temperature dependence of the epi-layer resistance is due to the temperature dependence of the low-field mobility of moderate doped n-type GaAs. The resistance $R_{s}\left(\mathrm{~T}_{\text {nom }}\right)$ in (9) 
is in general bias dependent but typically a fixed value extracted at a moderate to high forward bias can be used with acceptable results.

To illustrate the temperature dependence predicted by the developed model, the I-V characteristics for a triple anode stack are simulated at $77 \mathrm{~K}$ and at $300 \mathrm{~K}$, see Fig. 2. The Schottky barrier diodes are assumed to be similar to the diodes from Virginia Diodes Inc. used in the millimeter-wave multiplier discussed later. The measured I-V characteristic at $300 \mathrm{~K}$ is available for the triple anode stack. Using a few model parameters $\left(I_{s}, \eta, R_{s}\right.$ and $\left.C_{j o}\right)$ extracted at $300 \mathrm{~K}$ it is first verified, as shown in Fig. 2, that our developed SDD model predicts the measured characteristic at $300 \mathrm{~K}$. To predict the performance at cryogenic temperatures, representative values for the remaining model parameters are assumed. From the I-V characteristic at $77 \mathrm{~K}$ it is seen that the current level for a given voltage is lower and the slope is steeper than at $300 \mathrm{~K}$ in accordance with observations found in the literature [5]-[6].

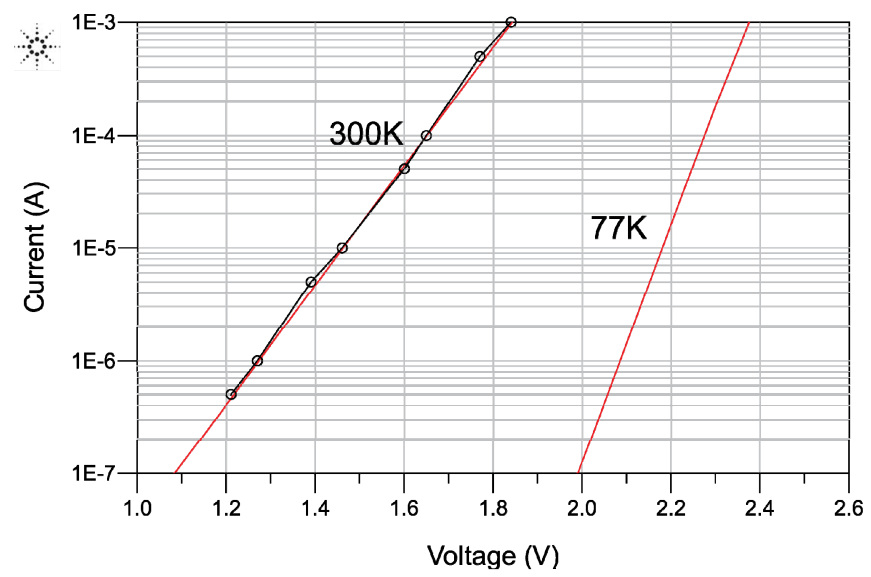

Fig. 2. I-V characteristics of three anode stack at $300 \mathrm{~K}$ and $77 \mathrm{~K}$. The measured data are shown as symbols. The assumed model parameters are: $I_{s}=1.8 \mathrm{e}-13 \mathrm{~A}, \eta=3.17, \mathrm{R}_{\mathrm{s}}=6.0 \Omega, \mathrm{X}_{\mathrm{r}}=0.6 \mathrm{C}_{\mathrm{jo}}=26 \mathrm{fF}, \mathrm{F}_{\mathrm{c}}=0.9, \quad{ }_{\mathrm{b}}=0.96$ $\mathrm{V}, \quad \mathrm{N}_{\mathrm{d}}=1 \mathrm{e} 17 \quad \mathrm{~cm}^{-3}, \quad \mathrm{~T}_{\text {nom }}=300 \mathrm{~K}, \quad \mathrm{R}_{\mathrm{th}}=500 \mathrm{~K} / \mathrm{W}, \quad \mathrm{C}_{\mathrm{th}}=1 \mathrm{e}-6 \mathrm{~J} / \mathrm{K}$, $\varepsilon_{\mathrm{s}}=13.1 \cdot 8.85 \mathrm{e}-12 \mathrm{~F} / \mathrm{m}, \alpha=2.2, \mathrm{~m}^{*}=0.068 \cdot 9.1 \mathrm{e}-31 \mathrm{~kg}, \mathrm{~N}_{\mathrm{c}}=4.37 \mathrm{e} 17 \mathrm{~cm}^{-3}$.

\section{DOUBLER CIRCUIT}

The model described above is used to evaluate the performance of doublers based on anti-series balanced topology (refer to Fig. 3). The advantage of the anti-series balanced configuration is suppression of odd order harmonics, while the amplitude of the second harmonic is double as high as for the case of an unbalanced single diode doubler. Three diodes are connected in series in each branch in order to enhance the power handling capability. Such an arrangement of diodes is equivalent to a single Schottky diode having triple as high reverse breakdown voltage. In anti-series configuration, diode arrays are fed out-of-phase by an input power source through a balancing transformer $T_{1}$. Turn ratio of the transformer is equal to 1 . A DC voltage source $V_{d}$ is used to bias the diodes. DC blocking capacitors $\mathrm{C}_{\mathrm{cl}}$ are placed at the output of the transformer to prevent DC current flowing into the transformer.
DC feeding inductors, $\mathrm{L}_{\mathrm{cl}}$, are used to isolate RF signal from DC voltage source and provide DC ground. The generator and load impedances $\left(Z_{g}\right.$ and $Z_{L}$ respectively) are frequency dependent and chosen to maximize doubler efficiency. $Z_{g}$ is conjugate matched to the input impedance of the doubler at frequency of the input signal, and represents a short circuit at $2^{\text {nd }}$ harmonic. The load impedance $Z_{\mathrm{L}}$, on the other hand, is conjugate matched at the $2^{\text {nd }}$ harmonic of the input signal, and represents a short circuit at the frequency of the input signal. In this analysis, the losses in the matching circuits are neglected. The diodes are reverse biased with negative $V_{d}$, which is chosen to maximize efficiency at an input power of $207 \mathrm{~mW}$.

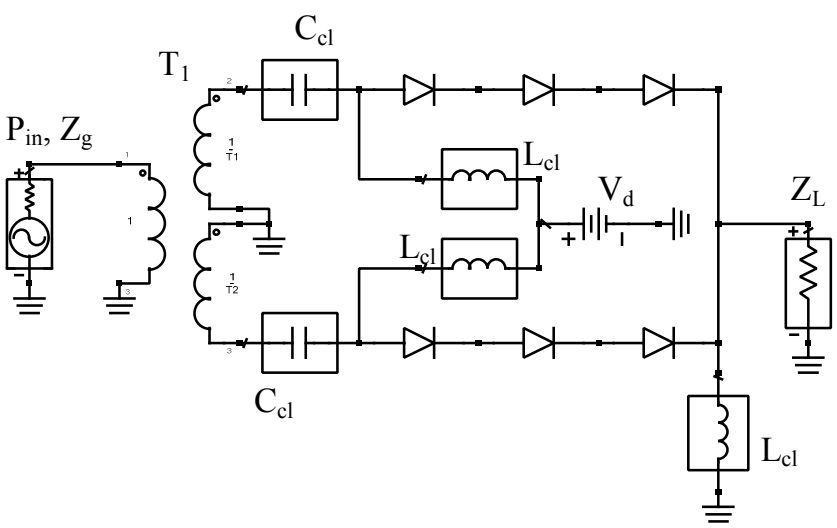

Fig. 3. Schematic of the circuit setup for harmonic balance simulation of frequency doublers in an anti-series configuration of diode array.

\section{MODEL VERIFICATION AGAINST MEASURED DATA}

The model developed in Section II has been used to evaluate the behavior of a $78 \mathrm{GHz}$ doubler described in [8]. This allows to test the model all the way down to $14 \mathrm{~K}$. The harmonic-balance analysis is applied to the circuit in Fig. 3 to theoretically estimate doubler efficiency at fixed input power of about $207 \mathrm{~mW}$ and frequency of interest $78 \mathrm{GHz}$. The graph in Fig. 4 shows estimated and measured efficiency versus temperature of the $78 \mathrm{GHz}$ doubler.

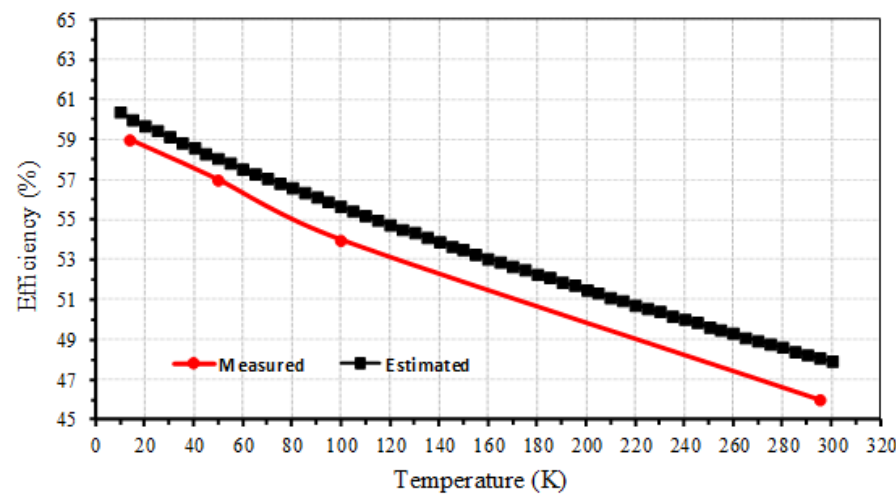

Fig. 4. Estimated and measured efficiency versus temperature of the $78 \mathrm{GHz}$ doubler. The measured data is extracted from [8]. 
As observed in Fig. 4, the overall behavior of the doubler is well predicted as the measured efficiency values are typically only $1-2 \%$ lower than the estimated ones. It is observed that the predicted efficiency can be greater than 60 $\%$ at $14 \mathrm{~K}$ temperature, which is comparable with the experimental result of $59 \%$ at the same temperature value. The minor discrepancy between estimated and measured results can partially be explained by the neglected losses in the matching circuit.

The developed Schottky barrier diode model allows us to investigate the reason for the larger multiplier efficiency at cryogenic temperatures by detailed simulations. For high efficiency millimeter-wave multipliers, the Schottky barrier diodes operate in varactor mode. It is thus mainly the nonlinear behavior of the $\mathrm{C}-\mathrm{V}$ characteristic that determines the efficiency of the multiplier. The non-linear $\mathrm{C}-\mathrm{V}$ characteristic, however, is found to vary little with temperature and can alone not explain the increase in efficiency at cryogenic temperatures. The main reason for the increased efficiency at lower temperature is found to be the increased maximum current of the varactor, when the doubler becomes cold. The maximum current is related to the resistance of the epi-layer which drops to a low value at cryogenic temperatures. Interestingly, the temperature dependence of the velocity saturation effect as described in [6] is shown to be of less importance for the considered millimeter-wave frequency doubler.

\section{CONCLUSIONS}

In this work, we have developed and evaluated a Schottky barrier diode model. The model was used to estimate the efficiency of a VDI doubler at $78 \mathrm{GHz}$ at cryogenic temperatures.
The excellent agreement with measured results shows that the model is suitable for predicting performance of millimeterwave multipliers at temperatures down to $14 \mathrm{~K}$, and, probably, below.

\section{ACKNOWLEDGMENT}

The authors would like to thank the Lundbeck Foundation for partial support of the activities.

\section{REFERENCES}

[1] J. H. Ardenkjær-Larsen, B. Fridlund, A. Gram, G. Hansson, L. Hansson, M. H. Lerche, R. Servin, M. Thaning, and K. Golman, "Increase in signal-to-noise ratio of $>10,000$ times in liquid-state NMR," National Academy of Sciences. Proceedings, vol. 100, pp. 10158-10163, 2003.

[2] L. Yan, Design of Integrated Circuits Approching Terahertz Frequencies, PhD Thesis, Technical University of Denmark, 2013.

[3] S. M. Sze and K. K. Ng, Physics of Semiconductor Devices, John Wiley \& Sons, $3^{\text {rd }}$ edition, 2007.

[4] L. Yan, V. Krozer, R. Michaelsen, T. Djurhuus, and T.K. Johansen, "Physical based Schottky barrier diode modeling for THz applications," in IEEE MTT International Wireless Symposium, Beijing, 2013.

[5] J. T. Louhi, A. Räisänen, and N. R. Erickson, "Cooled Schottky Varactor Frequency Multipliers at Submillimeter Wavelengths," IEEE Trans. Microw. Theory Techn., vol .41, no. 4, pp. 565-571, April 1993.

[6] E. L. Kollberg, H. Zirath, and A. Jelenski, "Temperature-Variable Characteristics and Noise in Metal-Semiconductor Junctions," IEEE Trans. Microw. Theory Techn., vol. 43, no.9, pp. 913-922, Sept. 1986.

[7] A.-Y. Tang et al., "Electro-Thermal Model for Multi-Anode Schottky Diode Multipliers", IEEE Trans. THz Sci. Technol., vol. 2, no. 3, pp. 290-298, May 2012.

[8] D. W. Porterfield, T. W. Crowe, R. F. Bradley, and N. R. Erickson, “A High-Power Fixed-Tuned Millimeter-Wave Balanced Frequency Doubler," IEEE Transactions on Microwave Theory and Techniques, Vol. 47, No. 4, pp. 419-425, April 1999. 